\title{
On the incompressible behavior in weakly nonlinear elasticity
}

Christopher Kube ( $\nabla$ kube@psu.edu )

The Pennsylvania State University https://orcid.org/0000-0002-1834-7040

\section{Research Article}

Keywords: nonlinear elasticity, fourth-order elastic constants, incompressibility

Posted Date: February 14th, 2022

DOI: https://doi.org/10.21203/rs.3.rs-1348072/v1

License: (9) This work is licensed under a Creative Commons Attribution 4.0 International License.

Read Full License

Version of Record: A version of this preprint was published at Journal of Elasticity on March 17th, 2022. See the published version at https://doi.org/10.1007/s10659-022-09886-4. 


\title{
On the incompressible behavior in weakly nonlinear elasticity
}

\author{
Christopher M. Kube
}

Received: February 10, 2022/ Accepted: date

\begin{abstract}
This article considers the influence of incompressibility on the compliance and stiffness constants that appear in the weakly nonlinear theory of elasticity. The formulation first considers the incompressibility constraint applied to compliances, which gives explicit finite limits for the second-, third-, and fourth-order compliance constants. The stiffness/compliance relationships for each order are derived and used to determine the incompressible behavior of the second-, third-, and fourth-order stiffness constants. Unlike the compressible case, the fourth-order compliances are not found to be dependent on the fourth-order stiffnesses.
\end{abstract}

Keywords nonlinear elasticity · fourth-order elastic constants · incompressibility

Mathematics Subject Classification (2000) 74B20 · 74B99 · 74A20

C.M. Kube

Engineering Science and Mechanics

Penn State University

University Park, PA 16802

E-mail: kube@psu.edu 


\section{$1 \quad 1$ Introduction}

2 The weakly nonlinear theory of elasticity applied to incompressible solids has renewed interest with growth in dynamic characterization of soft materials such as tissue. A recent article by Saccomandi and Vergori [1] recaps the historical developments and places the weakly nonlinear and nonlinear theories in an appropriate context. Thus, readers interested in a historical basis should consult Saccomandi and Vergori and the references therein [1]. However, the benefits of the weakly nonlinear theory are appropriate to highlight and restate. Namely, the weakly nonlinear theory of incompressible solids contains only three independent stiffness constants, including exactly one second-, third-, and fourth-order stiffness. Thus, weakly nonlinear elastodynamic models involving the three stiffnesses provides a straightforward bridge to experiments whereas the full nonlinear theory of elasticity often deals with functional dependencies requiring fitting of experimental data.

With this motivation, several researchers have investigated the behavior of elastic stiffnesses when constraints of incompressibility are applied within the weakly nonlinear theory. Kostek, Sinha, and Norris [2] deduced connections between the third-order stiffness constants $c_{111}, c_{112}$, and $c_{123}$ and the constants $A_{\ell}$ and $B_{\ell}$ present in the adiabatic equation of state for an inviscid fluid. In a similar comparison with liquids, Hamilton, Ilinskii, and Zabolotskaya obtained the behavior for the fourth-order stiffnesses $E, F, G$, and $H$ in terms of $A_{\ell}, B_{\ell}$, and $C_{\ell}$ in addition to a fourth-order constant $D_{\ell}[3]$. However, the comparison to a fluid does not lead to the possibility of shear deformation, which is a shortcoming if a connection to elastodynamic shear deformation in soft solids is desired. The existence of linear and nonlinear shear deformation was known theoretically for many years [4] and modern techniques involving the use of shear wave elastography are commonplace $[5,6]$. Destrade and Ogden uncovered the behavior of the linear and nonlinear stiffnesses under the constraints of incompressibility applied to the weakly nonlinear theory of compressible solids [7]. They were able to predict the magnitude of each of the stiffnessses to within the order of the Lamé parameter [7]. With the behavior of the stiffnesses known, the link between the strain energy of general compressible solids to that of an incompressible solid with three independent parameters was established as predicted decades before [4]. Additionally, other theoretical models developed for compressible solids could make use these results to provide the behavior as the solid tends toward incompressibility.

In Destrade and Ogden [7], the constraints stemming from incompressibility were applied to the relationship between logarithmic strain and the conjugate stress tensor (rather than the more common stress-strain relationship). In doing so, an arbitrary Lagrange multiplier is not needed in this case as stated in Destrade and Ogden, "...a strain-stress relation is more amenable to the imposition of incompressibility, because it leads to unambiguous, finite limit(s) for one (or several) compliance(s)" [7]. Thus, the analysis of the compliances are fundamental to determining the behavior in the incompressible limit. However, unlike in [8], which analyzed the behavior of the second-order compliance constants, the compliance constants and their behavior were not explicitly provided [7]. More recently, Saccomandi and Vergori [1] followed a similar approach of eliminating the Green-Lagrange strain invariants in the incompressibility constraint in favor

of invariants of the second Piola-Kirchhoff stress tensor. They obtained improved 
estimates of the Landau constants by then analyzing the stress-strain relationship including the determination of the Lagrange multiplier $p$. Once again, while the behavior of the compliance constants are integral to the method, their explicit behavior was not noted.

In this article, the limiting behavior of the second-, third-, and fourth-order elastic stiffnesses are obtained as the solid tends toward incompressibility. However, our approach makes explit and direct use of the second-, third-, and fourth-order compliance constants. Thus, within the analysis, the behavior of the compliances are reported for the first time. Additionally, the approach requires the use of stiffness/compliance relationships generally established using Brugger's convention [9] for the stiffnesses (involving $c_{12}, c_{44}, c_{123}, c_{144}, c_{456}, c_{1123}, c_{1255}, c_{1456}$ and $c_{4455}$ ) and compliances (involving $s_{12}, s_{44}, s_{123}, s_{144}, s_{456}, s_{1123}, s_{1255}, s_{1456}$ and $\left.s_{4455}\right)$. While conversions between the Landau constants and Brugger's constants are known and straightforward, the Brugger convention is more commonly used when analyzing anisotropic materials, which is a planned extension of this work. Furthermore, the results reported here in terms of Brugger's constants are expected to help extend theories formulated with Brugger's constants to the domain of incompressibility.

\section{Weakly nonlinear elasticity}

For a weakly nonlinear elastic solid, the second Piola-Kirchhoff stress tensor $\boldsymbol{S}$ can be written as a third-order polynomial in the Green-Lagrange strain $\boldsymbol{E}$,

$$
S_{i j}=c_{i j k l} E_{k l}+\frac{1}{2} c_{i j k l m n} E_{k l} E_{m n}+\frac{1}{6} c_{i j k l m n p q} E_{k l} E_{m n} E_{p q}
$$

where $c_{i j k l}, c_{i j k l m n}$, and $c_{i j k l m n p q}$ are tensors whose components are the second, third-, and fourth-order elastic stiffnesses, respectively. Equation (1) is given in general anisotropic form to motivate possible extensions of this work to anisotropic materials. The current article considers isotropic solids only for which $\boldsymbol{S}$ can be written as

$$
\begin{aligned}
\boldsymbol{S}= & {\left[c_{12} \mathcal{J}_{1}+\frac{1}{2} c_{123} \mathcal{J}_{1}^{2}+c_{144} \mathcal{J}_{2}+\frac{1}{6}\left(c_{1123}-2 c_{1255}+4 c_{1456}\right) \mathcal{J}_{1}^{3}\right.} \\
& \left.+\left(c_{1255}-2 c_{1456}\right) \mathcal{J}_{1} \mathcal{J}_{2}+\frac{4}{3} c_{1456} \mathcal{J}_{3}\right] \boldsymbol{\delta} \\
& +\left[2 c_{44}+2 c_{144} \mathcal{J}_{1}+\left(c_{1255}-2 c_{1456}\right) \mathcal{J}_{1}^{2}+2 c_{4455} \mathcal{J}_{2}\right] \boldsymbol{E} \\
& +4\left(c_{456}+c_{1456} \mathcal{J}_{1}\right) \boldsymbol{E}^{2}
\end{aligned}
$$

where the strain invariants are

$$
\begin{aligned}
\mathcal{J}_{1} & =\operatorname{tr} \boldsymbol{E} \\
\mathcal{J}_{2} & =\operatorname{tr} \boldsymbol{E}^{2} \\
\mathcal{J}_{3} & =\operatorname{tr} \boldsymbol{E}^{3} .
\end{aligned}
$$

The form of $\boldsymbol{S}$ in Eq. (2) is obtained through substituting the isotropic forms of $c_{i j k l}, c_{i j k l m n}$, and $c_{i j k l m n p q}$ given in Appendix A into Eq. (1) and evaluating terms involving Kronecker delta functions. Under a deformation, a compressible 
solid changes its differential volume according to $d V=\operatorname{det} \boldsymbol{F} d V_{0}$ where $\boldsymbol{F}$ is the deformation gradient. The quantity $\operatorname{det} \boldsymbol{F}$ is related to the principal invariants of the Green-Lagrange strain [2, 10],

$$
\operatorname{det} \boldsymbol{F}=\left(1+2 I_{E}+4 I I_{E}+8 I I I_{E}\right)^{1 / 2},
$$

where the principal invariants, and their relation to $\mathcal{J}_{1}, \mathcal{J}_{2}$, and $\mathcal{J}_{3}$, are

$$
\begin{aligned}
I_{E} & =\operatorname{tr} \boldsymbol{E}=\mathcal{J}_{1} \\
I I_{E} & =\frac{1}{2}\left(\operatorname{tr}^{2} \boldsymbol{E}-\operatorname{tr} \boldsymbol{E}^{2}\right)=\frac{1}{2}\left(\mathcal{J}_{1}^{2}-\mathcal{J}_{2}\right) \\
I I I_{E} & =\operatorname{det} \boldsymbol{E}=\frac{1}{6} \operatorname{tr}^{3} \boldsymbol{E}-\frac{1}{2} \operatorname{tr} \boldsymbol{E} \operatorname{tr} \boldsymbol{E}^{2}+\frac{1}{3} \operatorname{tr} \boldsymbol{E}^{3} \\
& =\frac{1}{6} \mathcal{J}_{1}^{3}-\frac{1}{2} \mathcal{J}_{1} \mathcal{J}_{2}^{2}+\frac{1}{3} \mathcal{J}_{3},
\end{aligned}
$$

Thus, in terms of the strain invariants $\mathcal{J}_{1}, \mathcal{J}_{2}$, and $\mathcal{J}_{3}$, Eq. 4 becomes

$$
\operatorname{det} \boldsymbol{F}=\left(1+2 \mathcal{J}_{1}+2 \mathcal{J}_{1}^{2}-2 \mathcal{J}_{2}+\frac{4}{3} \mathcal{J}_{1}^{3}-4 \mathcal{J}_{1} \mathcal{J}_{2}+\frac{8}{3} \mathcal{J}_{3}\right)^{1 / 2}
$$

A solid is incompressible when it is capable of only isochoric deformations, i.e., $\operatorname{det} \boldsymbol{F}=1$. This motivates forming the parameter $v$ from Eq. (6) in which $v \sim 0$ when $\operatorname{det} \boldsymbol{F}=1$,

$$
v=\frac{1}{2}\left[(\operatorname{det} \boldsymbol{F})^{2}-1\right]=\mathcal{J}_{1}+\mathcal{J}_{1}^{2}-\mathcal{J}_{2}+\frac{2}{3} \mathcal{J}_{1}^{3}-2 \mathcal{J}_{1} \mathcal{J}_{2}+\frac{4}{3} \mathcal{J}_{3} .
$$

Thus, the vanishing of $v$ based on the combinations of strain invariants seen in the right-hand side of Eq. (7) provides the desired constraint to analyze incompressibility. Now, as described by [7], transforming the Green-Lagrange strain in Eq. (7) to the second Piola-Kirchhoff stress circumvents the need for an arbitrary Lagrange multiplier. The transformation is provided through the strain-stress relationship,

$$
E_{i j}=s_{i j k l} S_{k l}+\frac{1}{2} s_{i j k l m n} S_{k l} S_{m n}+\frac{1}{6} s_{i j k l m n p q} S_{k l} S_{m n} S_{p q},
$$

where $s_{i j k l}, s_{i j k l m n}$, and $s_{i j k l m n p q}$ are tensors containing components of second-, third-, and fourth-order elastic compliances, respectively. Similar to Eq. (2), $\boldsymbol{E}$ for an isotropic solid takes the form

$$
\begin{aligned}
\boldsymbol{E}= & {\left[s_{12} \mathcal{S}_{1}+\frac{1}{2} s_{123} \mathcal{S}_{1}^{2}+s_{144} \mathcal{S}_{2}+\frac{1}{6}\left(s_{1123}-2 s_{1255}+4 s_{1456}\right) \mathcal{S}_{1}^{3}\right.} \\
& \left.+\left(s_{1255}-2 s_{1456}\right) \mathcal{S}_{1} \mathcal{S}_{2}+\frac{4}{3} s_{1456} \mathcal{S}_{3}\right] \boldsymbol{\delta} \\
& +\left[2 s_{44}+2 s_{144} \mathcal{S}_{1}+\left(s_{1255}-2 s_{1456}\right) \mathcal{S}_{1}^{2}+2 s_{4455} \mathcal{S}_{2}\right] \boldsymbol{S} \\
& +4\left(s_{456}+s_{1456} \mathcal{S}_{1}\right) \boldsymbol{S}^{2}
\end{aligned}
$$

where

$$
\begin{aligned}
& \mathcal{S}_{1}=\operatorname{tr} \boldsymbol{S} \\
& \mathcal{S}_{2}=\operatorname{tr} \boldsymbol{S}^{2} \\
& \mathcal{S}_{3}=\operatorname{tr} \boldsymbol{S}^{3},
\end{aligned}
$$


are invariants of the second Piola-Kirchhoff stress tensor. The next step involves obtaining the strain invariants needed in Eq. (7) by determining the trace of $\boldsymbol{E}$, $\boldsymbol{E}^{2}$, and $\boldsymbol{E}^{3}$ using Eq. (9), which leads to

$$
\begin{aligned}
\mathcal{J}_{1}= & \left(3 s_{12}+2 s_{44}\right) \mathcal{S}_{1}+\frac{1}{2}\left(3 s_{123}+4 s_{144}\right) \mathcal{S}_{1}^{2}+\left(3 s_{144}+4 s_{456}\right) \mathcal{S}_{2} \\
& +\frac{1}{2} s_{1123} \mathcal{S}_{1}^{3}+\left(3 s_{1255}-2 s_{1456}+2 s_{4455}\right) \mathcal{S}_{1} \mathcal{S}_{2}+4 s_{1456} \mathcal{S}_{3}+\mathcal{O}\left(\boldsymbol{S}^{4}\right) \\
\mathcal{J}_{2}= & s_{12}\left(3 s_{12}+4 s_{44}\right) \mathcal{S}_{1}^{2}+4 s_{44}^{2} \mathcal{S}_{2}+\left[\left(3 s_{12}+2 s_{44}\right) s_{123}+4 s_{12} s_{144}\right] \mathcal{S}_{1}^{3} \\
& +\left[6\left(s_{12}+2 s_{44}\right) s_{144}+8 s_{12} s_{456}\right] \mathcal{S}_{1} \mathcal{S}_{2}+16 s_{44} s_{456} \mathcal{S}_{3}+\mathcal{O}\left(\boldsymbol{S}^{4}\right) \\
\mathcal{J}_{3}= & 3 s_{12}^{2}\left(s_{12}+2 s_{44}\right) \mathcal{S}_{1}^{3}+12 s_{12} s_{44}^{2} \mathcal{S}_{1} \mathcal{S}_{2}+8 s_{44}^{3} \mathcal{S}_{3}+\mathcal{O}\left(\boldsymbol{S}^{4}\right) .
\end{aligned}
$$

Additionally, the following three quantities are needed in Eq. (7),

$$
\begin{aligned}
\mathcal{J}_{1}^{2}= & \left(3 s_{12}+2 s_{44}\right)^{2} \mathcal{S}_{1}^{2}+\left(3 s_{12}+2 s_{44}\right)\left(3 s_{123}+4 s_{144}\right) \mathcal{S}_{1}^{3} \\
& +2\left(3 s_{12}+2 s_{44}\right)\left(3 s_{144}+4 s_{456}\right) \mathcal{S}_{1} \mathcal{S}_{2}+\mathcal{O}\left(\boldsymbol{S}^{4}\right) \\
\mathcal{J}_{1}^{3}= & \left(3 s_{12}+2 s_{44}\right)^{3} \mathcal{S}_{1}^{3}+\mathcal{O}\left(\boldsymbol{S}^{4}\right) \\
\mathcal{J}_{1} \mathcal{J}_{2}= & s_{12}\left(3 s_{12}+2 s_{44}\right)\left(3 s_{12}+4 s_{44}\right) \mathcal{S}_{1}^{3}+4 s_{44}^{2}\left(3 s_{12}+2 s_{44}\right) \mathcal{S}_{1} \mathcal{S}_{2}+\mathcal{O}\left(\boldsymbol{S}^{4}\right) .
\end{aligned}
$$

Truncating Eqs. (11)-(12) to include up to third-order terms involving $\boldsymbol{S}$ and substituting into Eq. (7) gives

$$
v=a_{1} \mathcal{S}_{1}+a_{2} \mathcal{S}_{2}+\frac{1}{2} a_{3} \mathcal{S}_{1}^{2}+a_{4} \mathcal{S}_{1} \mathcal{S}_{2}+\frac{1}{2} a_{5} \mathcal{S}_{1}^{3}+\frac{4}{3} a_{6} \mathcal{S}_{3},
$$

where

$$
\begin{aligned}
a_{1}= & 3 s_{12}+2 s_{44}, \\
a_{2}= & 3 s_{144}+4 s_{456}-4 s_{44}^{2}, \\
a_{3}= & 3 s_{123}+4 s_{144}+\frac{4}{3}\left(a_{1}^{2}+2 s_{44}^{2}\right), \\
a_{4}= & 3 s_{1255}-2 s_{1456}+2 s_{4455}+\frac{4}{3}\left(a_{1}-2 s_{44}\right)\left(3 s_{144}+4 s_{456}-2 s_{44}^{2}\right) \\
& +16 s_{44}\left(s_{456}-s_{44}^{2}\right), \\
a_{5}= & s_{1123}+4 a_{1} s_{123}+\frac{16}{3} a_{1} s_{144}+\frac{16}{3} s_{44} s_{144}+\frac{16}{9} a_{1} s_{44}^{2}+\frac{8}{27} a_{1}^{3}+\frac{128}{27} s_{44}^{3}, \\
a_{6}= & 3 s_{1456}-12 s_{44} s_{456}+8 s_{44}^{3} .
\end{aligned}
$$

An expression similar to Eq. (13), but not equivalent, was given by Saccomandi and Vergori [1]. In Eq. (14), the $a_{i}$ coefficients are functions of the second-, third-, and fourth-order compliance constants. Previous work [1, 7] made use of inverting the strain invariants into stress invariants, but did not explicitly involve or make use of the compliance constants. The advantage of using compliance constants includes a logical path forward to investigate anisotropic materials and also provides new insights into the behavior of compliances when solids tend toward incompressibility. 


\section{Behavior of nonlinear compliance constants in the incompressible} limit

The behavior of the compliance constants seen in Eq. (14) as a solid tends toward incompresibility are obtained by observing the behavior of $v \sim 0$ as each coefficient $a_{i}$ vanishes. The vanishing of each constant $a_{i}$ are necessary and sufficient conditions for $v \sim 0$ [1]. For an isotropic solid, the compliance constants $s_{44}$ and $s_{456}$ are functions of only the shear stiffnesses $s_{44}=\left(4 c_{44}\right)^{-1}$ and $s_{456}=-c_{456}\left(8 c_{44}^{3}\right)^{-1}$, which were previously proven to remain finite $[1,3,7]$. Thus, the constants $s_{12}$, $s_{144}$ and $s_{123}$ can be solved in terms of $s_{44}$ and $s_{456}$ after letting $a_{1}, a_{2}$, and $a_{3}$ vanish,

$$
\begin{aligned}
s_{12} & \sim-\frac{2}{3} s_{44} \\
s_{144} & \sim \frac{4}{3} s_{44}^{2}-\frac{4}{3} s_{456} \\
s_{123} & \sim-\frac{4}{9} a_{1}^{2}-\frac{8}{3} s_{44}^{2}+\frac{16}{9} s_{456} .
\end{aligned}
$$

Equations (15) provide the complete behavior of the second-, and third-order compliances in the incompressible limit. It is interesting to note that all of the compliances remain finite, whereas only the stiffnesses $c_{44}$ and $c_{456}$ remain finite.

To examine the behavior of the fourth-order compliances, we begin by analyzing the expression involving $a_{6}$ to give

$$
s_{1456} \sim-\frac{8}{3} s_{44}^{3}+4 s_{44} s_{456}
$$

The behavior of $s_{1123}$ is obtained from the $a_{5}$ expression together with $s_{123}$ and $s_{144}$ from Eq. (15),

$$
s_{1123} \sim \frac{40}{27} a_{1}^{3}+\frac{16}{9} a_{1} s_{44}^{2}-\frac{320}{27} s_{44}^{3}+\frac{64}{9} s_{44} s_{456} .
$$

Lastly, a similar analysis involving the $a_{4}$ expression leads to

$$
s_{1255} \sim-\frac{8}{9} a_{1} s_{44}^{2}+\frac{16}{3} s_{44}^{3}-\frac{8}{3} s_{44} s_{456}-\frac{2}{3} s_{4455} .
$$

Note that each term multiplying factors of $a_{1}$ in Eqs. (15)-(18) are finite and vanish as $a_{1} \sim 0$. The choice to include the $a_{1}$ factors are because they will be needed in the evaluation of the stiffnesses in Sec. 4. A summary of the behavior of the compliance constants in terms of the stiffnesses $c_{44}$ and $c_{456}$ is given in Sec. 5 .

\section{Behavior of nonlinear stiffness constants in the incompressible limit}

To investigate the behavior of the stiffness constants under constraints of incompressibility, we begin by analyzing the linear elastic case involving $a_{1}$ in Eq. (14). Using stiffness/compliance relations, $a_{1}$ can be written as

$$
a_{1}=\frac{1}{3 c_{12}+2 c_{44}} \text {. }
$$


Thus, the vanishing of $a_{1}$ leads to $c_{12} \sim \infty$ and $a_{1} \sim 1 /\left(3 c_{12}\right)$.

To find the behavior of the third- and fourth-order stiffnesses, extensive use is made of the stiffness/compliance relationships found in Appendix B, which were derived with no consideration of incompressibility. For $c_{144}$, the third-order stiffness/compliance relationship for $s_{144}$ is rewritten as the expression

$$
0=s_{144}+\frac{a_{1}}{12 c_{44}^{2}}\left(3 c_{144}+4 c_{456}\right)-\frac{c_{456}}{6 c_{44}^{3}},
$$

which holds in general. Now, Eq. (15) provides $s_{144} \sim-4 s_{456} / 3+4 s_{44}^{2} / 3=$ $c_{456} /\left(6 c_{44}^{3}\right)+1 /\left(12 c_{44}^{2}\right)$ where we have made use of the relationships, $s_{44}=1 /\left(4 c_{44}\right)$ and $s_{456}=-8 c_{456} s_{44}^{3}=-c_{456} /\left(8 c_{44}^{3}\right)$. Thus, after straightforward simplification, Eq. (20) becomes

$$
0=\frac{1}{12 c_{44}^{2}}\left(3 a_{1} c_{144}+4 a_{1} c_{456}+1\right),
$$

which, with $c_{44}$ and $c_{456}$ being finite, requires $3 a_{1} c_{144} \sim-1$ or $c_{144} \sim-1 /\left(3 a_{1}\right) \sim$ $-c_{12} \sim-\infty$.

Now, consider the stiffness/compliance relationship for $s_{123}$,

$$
0=s_{123}+a_{1}^{3}\left(c_{123}+2 c_{144}+\frac{8}{9} c_{456}\right)-\frac{a_{1}}{6 c_{44}^{2}}\left(3 c_{144}+4 c_{456}\right)+\frac{2 c_{456}}{9 c_{44}^{3}} .
$$

Using the result $s_{123} \sim-4 a_{1}^{2} / 9-8 s_{44}^{2} / 3+16 s_{456} / 9=-4 a_{1}^{2} / 9-2 c_{456} /\left(9 c_{44}^{3}\right)-$ $1 /\left(6 c_{44}^{2}\right)$ from Eq. (15) and $c_{144} \sim-1 /\left(3 a_{1}\right)$ leads to

$$
0=a_{1}^{3} c_{123}-\frac{1}{6 c_{44}^{2}}\left(3 a_{1} c_{144}+4 a_{1} c_{456}+1\right) .
$$

The second term in Eq. (23) must be zero because of Eq. (21) and, thus, $c_{123} \sim$ $\mathcal{O}\left(1 / a_{1}^{m}\right) \sim \mathcal{O}\left(c_{12}^{m}\right)$ where $m<3$ and the sign is unknown. An expression similar to Eq. (23) was derived previously by Destrade and Ogden [see their Eq. (26)] [7], but they concluded that $c_{123} \sim \mathcal{O}\left(c_{12}^{2}\right)$. Clearly, the present theory includes the possibility of $m=2$. However, the authors believe the exponent $m$ is likely material dependent.

Now, consider the behavior of the fourth-order elastic stiffnesses in a similar manner. Start with the stiffness/compliance relation involving $s_{1456}$,

$$
0=s_{1456}+\frac{a_{1}}{c_{44}}\left[\frac{c_{1456}}{8 c_{44}}+s_{456}\left(3 c_{144}+4 c_{456}\right)\right] .
$$

With $c_{144} \sim-1 /\left(3 a_{1}\right)$ and $s_{1456} \sim-\left(c_{44}+3 c_{456}\right) /\left(24 c_{44}^{4}\right)$, Eq. (24) becomes

$$
0 \sim 3 a_{1} c_{1456}-1 \text {. }
$$

Thus, $c_{1456} \sim 1 /\left(3 a_{1}\right) \sim c_{12} \sim \infty$. Next, consider the expression involving $s_{4455}$,

$$
0=s_{4455}+\frac{c_{4455}}{16 c_{44}^{4}}+\frac{1}{4 c_{44}^{2}}\left[c_{144}\left(3 s_{144}+4 s_{456}\right)+4 c_{456}\left(s_{144}+2 s_{456}\right)\right] .
$$

Substituting $c_{144} \sim-1 /\left(3 a_{1}\right), 3 s_{144} \sim-4 s_{456}+1 /\left(4 c_{44}^{2}\right)=\left(c_{44}+2 c_{456}\right) /\left(4 c_{44}^{3}\right)$, and $s_{456}=-c_{456} /\left(8 c_{44}^{3}\right)$ gives

$$
0=s_{4455}+\frac{c_{4455}}{16 c_{44}^{4}}+\frac{c_{456}}{12 c_{44}^{5}}\left(c_{44}-c_{456}\right)-\frac{1}{48 a_{1} c_{44}^{4}} .
$$


The $s_{4455}$ term is presumed finite and, thus, $s_{4455} \sim-c_{456}\left(c_{44}-c_{456}\right) /\left(12 c_{44}^{5}\right)$ and $c_{4455} \sim 1 /\left(3 a_{1}\right) \sim c_{12} \sim \infty$. The next term to consider involves $s_{1255}$,

$$
\begin{aligned}
0= & s_{1255}+\frac{4}{3} a_{1}^{2}\left[\frac{1}{16 c_{44}^{2}}\left(3 c_{1255}+2 c_{1456}+2 c_{4455}\right)\right. \\
& \left.-\frac{1}{12}\left(3 s_{144}+4 s_{456}\right)\left(9 c_{123}+18 c_{144}+8 c_{456}\right)\right] \\
& -\frac{4 a_{1}}{3 c_{44}}\left[\frac{c_{1456}}{16 c_{44}^{2}}-\frac{1}{6}\left(3 s_{144}-s_{456}\right)\left(3 c_{144}+4 c_{456}\right)\right] \\
& -\frac{c_{4455}}{24 c_{44}^{4}}-\frac{1}{18 c_{44}^{2}}\left[3 c_{144}\left(3 s_{144}+4 s_{456}\right)+8 c_{456} s_{456}\right] .
\end{aligned}
$$

Using the relationships $c_{144} \sim-1 /\left(3 a_{1}\right), c_{4455} \sim 1 /\left(3 a_{1}\right), c_{1456} \sim 1 /\left(3 a_{1}\right), s_{144} \sim$ $\left(c_{44}+2 c_{456}\right) /\left(12 c_{44}^{3}\right), s_{1255} \sim-2 s_{4455} / 3+8 s_{44} s_{456} / 3 \sim-c_{456}\left(c_{44}+2 c_{456}\right) /\left(36 c_{44}^{5}\right)$ leads to

$$
0=a_{1}^{2}\left(c_{123}+c_{1255}\right) .
$$

Now, recall that $c_{123} \sim \mathcal{O}\left(1 / a_{1}^{m}\right) \sim \mathcal{O}\left(c_{12}^{m}\right)$ with $m<3$ and, thus, $c_{1255} \sim$ $-c_{123} \sim-\mathcal{O}\left(1 / a_{1}^{m}\right) \sim-\mathcal{O}\left(c_{12}^{m}\right)$. Lastly, the stiffness/compliance relationships involving $s_{1123}$ can be used to find the behavior of $c_{1123}$,

4
7

7
7

$$
\begin{aligned}
0= & s_{1123}+a_{1}^{4}\left(c_{1123}+2 c_{1255}+\frac{4}{3} c_{4455}-\frac{4}{9} c_{1456}\right) \\
& -\frac{a_{1}^{2}}{3}\left[\frac{1}{2 c_{44}^{2}}\left(3 c_{1255}+2 c_{4455}+2 c_{1456}\right)\right. \\
& \left.-\left(3 s_{123}+4 s_{144}\right)\left(9 c_{123}+18 c_{144}+8 c_{456}\right)\right] \\
& -\frac{4 a_{1}}{3 c_{44}}\left[s_{144}\left(3 c_{144}+4 c_{456}\right)-\frac{1}{6} c_{1456}\right] .
\end{aligned}
$$

Using the relationships $c_{144} \sim-1 /\left(3 a_{1}\right), c_{4455} \sim 1 /\left(3 a_{1}\right), c_{1456} \sim 1 /\left(3 a_{1}\right), s_{144} \sim$ $\left(c_{44}+2 c_{456}\right) /\left(12 c_{44}^{3}\right), s_{1123} \sim 40 a_{1}^{3} / 27+16 a_{1} s_{44}^{2} / 9-320 s_{44}^{3} / 27+64 s_{44} s_{456} / 9=$ $40 a_{1}^{3} / 27+a_{1} /\left(9 c_{44}^{2}\right)-5 /\left(27 c_{44}^{3}\right)-2 c_{456} /\left(9 c_{44}^{4}\right)$, Eq. (30) becomes

$$
0=a_{1}^{4} c_{1123}
$$

\footnotetext{
Thus, $c_{1123} \sim \mathcal{O}\left(1 / a_{1}^{n}\right) \sim \mathcal{O}\left(c_{12}^{n}\right)$ with $n<4$ for Eq. (31) to be satisfied.
} 


\section{Summary of results}

Here, the results from Secs. 4 and 5 are recapped and organized. The behavior of the compliance constants as the solid tends to incompressibility are

$$
\begin{aligned}
& s_{12} \sim-\frac{2}{3} s_{44}=-\frac{1}{6 c_{44}}, s_{44}=\frac{1}{4 c_{44}}, \\
& s_{123} \sim-\frac{8}{3} s_{44}^{2}+\frac{16}{9} s_{456}=-\frac{1}{18 c_{44}^{3}}\left(3 c_{44}+4 c_{456}\right), \\
& s_{144} \sim \frac{4}{3} s_{44}^{2}-\frac{4}{3} s_{456}=\frac{1}{12 c_{44}^{3}}\left(c_{44}+2 c_{456}\right), s_{456}=-\frac{c_{456}}{8 c_{44}^{3}}, \\
& s_{1123} \sim-\frac{320}{27} s_{44}^{3}+\frac{64}{9} s_{44} s_{456}=-\frac{1}{27 c_{44}^{4}}\left(5 c_{44}+6 c_{456}\right), \\
& s_{1255} \sim \frac{16}{3} s_{44}^{3}-\frac{40}{9} s_{44} s_{456}-\frac{8 s_{456}^{2}}{9 s_{44}}=\frac{1}{36 c_{44}^{5}}\left(3 c_{44}^{2}+5 c_{44} c_{456}-2 c_{456}^{2}\right), \\
& s_{1456} \sim 4 s_{44} s_{456}-\frac{8}{3} s_{44}^{3}=-\frac{1}{24 c_{44}^{4}}\left(c_{44}+3 c_{456}\right), \\
& s_{4455} \sim \frac{4}{3 s_{44}}\left(2 s_{44}^{2}+s_{456}\right)=-\frac{c_{456}}{12 c_{44}^{5}}\left(c_{44}-c_{456}\right),
\end{aligned}
$$

where the general stiffness/compliance constants were used to convert the formulas to involve only stiffnesses. It is interesting, but perhaps expected, that all compliance constants remain finite and work out to be functions of the two shear stiffnesses $c_{44}$ and $c_{456}$. Furthermore, the fourth-order compliance constants are functions of fourth-order stiffnesses when the material is compressible, whereas this dependency is lost when incompressibility is applied.

The elastic stiffnesses were analyzed in Sec. 4 where the following results were observed

$$
\begin{aligned}
& c_{12} \sim \infty, c_{44} \sim \mathcal{O}(1), c_{123} \sim \mathcal{O}\left(c_{12}^{m}\right), c_{144} \sim-c_{12}, c_{456} \sim \mathcal{O}(1), \\
& c_{1123} \sim \mathcal{O}\left(c_{12}^{n}\right), c_{1255}=-c_{123} \sim-\mathcal{O}\left(c_{12}^{m}\right), c_{1456} \sim c_{12}, c_{4455} \sim c_{12}
\end{aligned}
$$

with $m<3$ and $n<4$. The stiffneses in Eq. (33) are presented using Brugger's convention. The conversion to the Landau-Lifshitz notation is completed using formulas in Appendix A and give

$$
\begin{aligned}
& \lambda \sim \infty, \mu \sim \mathcal{O}(1), C \sim \mathcal{O}\left(\lambda^{m}\right), B \sim-\lambda, A \sim \mathcal{O}(1), \\
& H \sim \frac{1}{24} \mathcal{O}\left(\lambda^{n}\right)+\frac{1}{12} \mathcal{O}\left(\lambda^{m}\right)+\frac{1}{6} \lambda, G \sim \frac{1}{2} \lambda, \quad F \sim-\mathcal{O}\left(\lambda^{m}\right)-\lambda, E \sim \frac{4}{3} \lambda
\end{aligned}
$$

where $m<3$ and $n<4$. Improved estimates for the magnitudes of $c_{123}, c_{1255}$, and $c_{1123}$ are possible by analyzing specific phenomenon. For example, Destrade and Ogden showed that if $c_{123}$ were linear in $c_{12}$, then the theory would predict a non-physical longitudinal wave mode [7]. Additionally, Saccomandi and Vergori obtained further information by analyzing the stress-strain relation including the Lagrange multiplier. Their results find improved limits on $m$ and $n$ such that these quantities obey $m<2$ and $n<3$. 


\section{Acoustoelasticity}

Previous researchers [1, 7] provide results for various nonlinear wave phenomenon using the obtained behavior of the stiffnesses in the incompressible limit. Thus, those results are not repeated here. However, it is worth noting an interesting connection between acoustoelasticity and the compliance constants. The shear phase velocity $v_{T}$ of a wave propagating in direction $\hat{\mathbf{n}}$ and displacement $\hat{\mathbf{u}}$ in an incompressible solid containing the static stress $\boldsymbol{\sigma}$ is governed by the expression

$$
\rho v_{T}^{2}=c_{44}-\left(1+\frac{2 c_{456}}{3 c_{44}}\right) \operatorname{tr} \boldsymbol{\sigma}+\left(2+\frac{c_{456}}{c_{44}}\right) \hat{\mathbf{n}} \cdot \boldsymbol{\sigma} \hat{\mathbf{n}}+\left(1+\frac{c_{456}}{c_{44}}\right) \hat{\mathbf{u}} \cdot \boldsymbol{\sigma} \hat{\mathbf{u}}
$$

which is a generalized form for the specific cases reported in [7]. It is interesting that the fourth-order compliances $s_{1123}, s_{1144}, s_{1456}$, and $s_{4455}$ are functions of only $c_{44}$ and $c_{456}$. Thus, acoustoelastic measurements provide direct access to these fourth-order compliances.

\section{Conclusion}

In this article, the incompressible behavior of both compliances and stiffnesses are derived explicitly. The second-, third-, and fourth-order compliance constants are shown to be finite. Additionally, the third- and fourth-order compliances are found to be functions of the finite stiffnesses $c_{44}$ and $c_{456}$, but not fourth-order stiffnesses. Thus, experiments sensitive to $c_{44}$ and $c_{456}$ can be connected to the fourth-order compliance constants. Lastly, the results are presented in both the Brugger convention [9] and Landau-Lifshitz notation [11]. Formulating the theory using the Brugger convention was necessary as the stiffness/compliance relationships given in Appendix B were obtained through tensorial operations. Furthermore, the Brugger convention is more commonly used in treating anisotropic problems, which provides a path forward to investigating the incompressible behavior of anisotropic materials. 


\section{Appendix A: Isotropic tensors of elastic stiffness and compliance}

For isotropic materials, the elastic modulus tensors are linear combinations of products of Kronecker delta functions,

$$
\begin{aligned}
& c_{i j k l}=c_{12} \delta_{i j} \delta_{k l}+2 c_{44} I_{i j k l}, \\
& c_{i j k l m n}=c_{123} \delta_{i j} \delta_{k l} \delta_{m n}+2 c_{144}\left(\delta_{i j} I_{k l m n}+\delta_{k l} I_{i j m n}+\delta_{m n} I_{i j k l}\right) \\
&+ 2 c_{456}\left(\delta_{i n} I_{j m k l}+\delta_{j n} I_{i m k l}+\delta_{i m} I_{j n k l}+\delta_{j m} I_{i n k l}\right), \\
& c_{i j k l m n p q}=\left(c_{1123}-2 c_{1255}+4 c_{1456}\right) \delta_{i j} \delta_{k l} \delta_{m n} \delta_{p q} \\
&+2\left(c_{1255}-2 c_{1456}\right)\left(\delta_{i j} \delta_{k l} I_{m n p q}+\delta_{i j} \delta_{m n} I_{k l p q}+\delta_{i j} \delta_{p q} I_{k l m n}\right. \\
&\left.+\delta_{k l} \delta_{m n} I_{i j p q}+\delta_{k l} \delta_{p q} I_{i j m n}+\delta_{m n} \delta_{p q} I_{i j k l}\right) \\
&+2 c_{1456}\left[\delta_{i j}\left(\delta_{k q} I_{l p m n}+\delta_{k p} I_{l q m n}+\delta_{l q} I_{k p m n}+\delta_{l p} I_{k q m n}\right)\right. \\
&+\delta_{k l}\left(\delta_{i q} I_{j p m n}+\delta_{i p} I_{j q m n}+\delta_{j q} I_{i p m n}+\delta_{j p} I_{i q m n}\right) \\
&+\delta_{m n}\left(\delta_{i q} I_{j p k l}+\delta_{i p} I_{j q k l}+\delta_{j q} I_{i p k l}+\delta_{j p} I_{i q k l}\right) \\
&\left.+\delta_{p q}\left(\delta_{i n} I_{j m k l}+\delta_{i m} I_{j n k l}+\delta_{j n} I_{i m k l}+\delta_{j m} I_{i n k l}\right)\right] \\
&+4 c_{4455}\left(I_{i j k l} I_{m n p q}+I_{i j m n} I_{k l p q}+I_{i j p q} I_{k l m n}\right)
\end{aligned}
$$

where $I_{i j k l}=\left(\delta_{i k} \delta_{j l}+\delta_{i l} \delta_{j k}\right) / 2$ is the fourth-rank identity tensor. The form of $c_{i j k l}$ is well-known whereas the forms for $c_{i j k l m n}$ and $c_{i j k l m n p q}$ are less common. Using Eqs. (36), it is easy to show that [12] $c_{11}=c_{12}+2 c_{44}, c_{111}=c_{123}+$ $6 c_{144}+8 c_{456}, c_{112}=c_{123}+2 c_{144}, c_{155}=c_{144}+2 c_{456}, c_{1111}=c_{1123}+10 c_{1255}+$ $12 c_{1456}+12 c_{4455}, c_{1112}=c_{1123}+4 c_{1255}, c_{1155}=c_{1255}+2 c_{1456}+2 c_{4455}$. Note that the analgous isotropic tensors for compliance constants take the same form as Eqs. (36), thus, only a notation change from $c \rightarrow s$ is needed.

Conversions to Landau-Lifshitz notation follow[10-12] $c_{11}=\lambda+2 \mu, c_{12}=\lambda$, $c_{44}=\mu, c_{111}=2 \mathcal{A}+6 \mathcal{B}+2 \mathcal{C}, c_{112}=2 \mathcal{B}+2 \mathcal{C}, c_{123}=2 \mathcal{C}, c_{144}=\mathcal{B}, c_{155}=$ $\mathcal{A} / 2+\mathcal{B}, c_{456}=\mathcal{A} / 4, c_{1111}=24(\mathcal{E}+\mathcal{F}+\mathcal{G}+\mathcal{H}), c_{1112}=6 \mathcal{E}+12 \mathcal{F}+24 \mathcal{H}$, $c_{1122}=8 \mathcal{F}+8 \mathcal{G}+24 \mathcal{H}, c_{1123}=4 \mathcal{F}+24 \mathcal{H}, c_{1255}=3 \mathcal{E} / 2+2 \mathcal{F}, c_{1456}=3 \mathcal{E} / 4$, $c_{1144}=2 \mathcal{F}+4 \mathcal{G}, c_{1155}=3 \mathcal{E}+2 \mathcal{F}+4 \mathcal{G}, c_{1244}=3 \mathcal{E} / 2+2 \mathcal{F}, c_{1266}=3 \mathcal{E}+2 \mathcal{F}$, $c_{4455}=2 \mathcal{G}, c_{4444}=6 \mathcal{G}$. Thus, in terms of the [11] convention,

$$
\begin{aligned}
c_{i j k l}=\lambda \delta_{i j} & \delta_{k l}+2 \mu I_{i j k l} \\
c_{i j k l m n}=2 \mathcal{C} & \delta_{i j} \delta_{k l} \delta_{m n}+2 \mathcal{B}\left(\delta_{i j} I_{k l m n}+\delta_{k l} I_{i j m n}+\delta_{m n} I_{i j k l}\right) \\
+ & \frac{\mathcal{A}}{4}\left(\delta_{i n} I_{j m k l}+\delta_{j n} I_{i m k l}+\delta_{i m} I_{j n k l}+\delta_{j m} I_{i n k l}\right) \\
c_{i j k l m n p q}= & 24 \mathcal{H} \delta_{i j} \delta_{k l} \delta_{m n} \delta_{p q}+4 \mathcal{F}\left(\delta_{i j} \delta_{k l} I_{m n p q}+\delta_{i j} \delta_{m n} I_{k l p q}+\delta_{i j} \delta_{p q} I_{k l m n}\right. \\
& \left.+\delta_{k l} \delta_{m n} I_{i j p q}+\delta_{k l} \delta_{p q} I_{i j m n}+\delta_{m n} \delta_{p q} I_{i j k l}\right) \\
& +\frac{3}{2} \mathcal{E}\left[\delta_{i j}\left(\delta_{k q} I_{l p m n}+\delta_{k p} I_{l q m n}+\delta_{l q} I_{k p m n}+\delta_{l p} I_{k q m n}\right)\right. \\
& +\delta_{k l}\left(\delta_{i q} I_{j p m n}+\delta_{i p} I_{j q m n}+\delta_{j q} I_{i p m n}+\delta_{j p} I_{i q m n}\right) \\
& +\delta_{m n}\left(\delta_{i q} I_{j p k l}+\delta_{i p} I_{j q k l}+\delta_{j q} I_{i p k l}+\delta_{j p} I_{i q k l}\right) \\
& \left.+\delta_{p q}\left(\delta_{i n} I_{j m k l}+\delta_{i m} I_{j n k l}+\delta_{j n} I_{i m k l}+\delta_{j m} I_{i n k l}\right)\right] \\
& +8 \mathcal{G}\left(I_{i j k l} I_{m n p q}+I_{i j m n} I_{k l p q}+I_{i j p q} I_{k l m n}\right) .
\end{aligned}
$$


Appendix B: Stiffness/compliance relationships

The derivative of $\boldsymbol{S}$ defined in Eq. 1 with respect to itself is

$$
\frac{\partial S_{i j}}{\partial S_{k l}}=I_{i j k l}=c_{i j m n} \frac{\partial E_{m n}}{\partial S_{k l}}+c_{i j m n p q} \frac{\partial E_{m n}}{\partial S_{k l}} E_{p q}+\frac{1}{2} c_{i j m n p q r s} \frac{\partial E_{m n}}{\partial S_{k l}} E_{p q} E_{r s},
$$

where $I_{i j k l}=\left(\delta_{i k} \delta_{j l}+\delta_{i l} \delta_{j k}\right) / 2$ is the fourth-rank identity. The term $\partial E_{m n} / \partial S_{k l}$ is found by taking the derivative of $\boldsymbol{E}$ in Eq. (8) with respect to $\boldsymbol{S}$,

$$
\frac{\partial E_{m n}}{\partial S_{k l}}=s_{m n k l}+s_{m n k l p q} S_{p q}+\frac{1}{2} s_{m n k l p q r s} S_{p q} S_{r s}
$$

which was obtained by using $\partial S_{i j} / \partial S_{k l}=I_{i j k l}$. Substituting Eqs. (8) and (39) into Eq. (38) and keeping terms up to second-order in $\boldsymbol{S}$ gives

$$
\begin{aligned}
I_{i j k l}= & c_{i j m n} s_{m n k l}+c_{i j m n} s_{m n k l p q} S_{p q}+c_{i j m n r s} s_{m n k l} s_{r s p q} S_{p q}+\frac{1}{2} c_{i j m n} s_{m n k l p q r s} S_{p q} S_{r s} \\
& +\frac{1}{2} c_{i j m n t u} s_{m n k l} s_{t u p q r s} S_{p q} S_{r s}+c_{i j m n t u} s_{m n k l p q} s_{t u r s} S_{p q} S_{r s} \\
& +\frac{1}{2} c_{i j m n t u x y} s_{m n k l} s_{t u p q} s_{x y r s} S_{p q} S_{r s}
\end{aligned}
$$

The connections between the elastic stiffness and compliance tensors are found by equating constant, linear, and quadratic terms of $\boldsymbol{S}$,

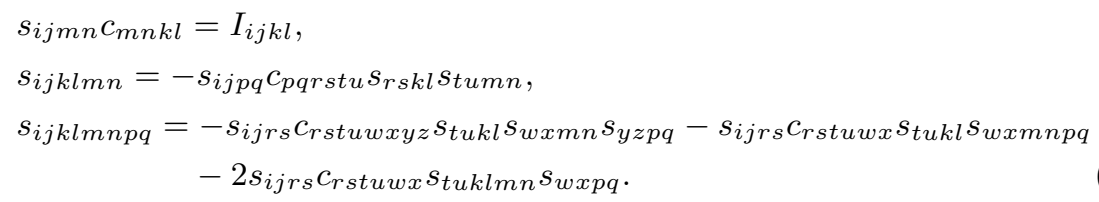


The stiffness/compliance relations for isotropic materials follow from Eqs. (41),

$$
\begin{aligned}
s_{12}=- & \frac{c_{12}}{2 c_{44}\left(3 c_{12}+2 c_{44}\right)}, s_{44}=\frac{1}{4 c_{44}}, \\
s_{123}=- & a_{1}^{3}\left(c_{123}+2 c_{144}+\frac{8}{9} c_{456}\right)+\frac{8}{3} a_{1} s_{44}^{2}\left(3 c_{144}+4 c_{456}\right)-\frac{128}{9} s_{44}^{3} c_{456}, \\
s_{144}=- & \frac{4}{3} a_{1} s_{44}^{2}\left(3 c_{144}+4 c_{456}\right)+\frac{32}{3} s_{44}^{3} c_{456}, s_{456}=-8 c_{456} s_{44}^{3}, \\
s_{1123}=- & a_{1}^{4}\left(c_{1123}+2 c_{1255}+\frac{4}{3} c_{4455}-\frac{4}{9} c_{1456}\right) \\
& +\frac{1}{3} a_{1}^{2}\left[8 s_{44}^{2}\left(3 c_{1255}+2 c_{4455}+2 c_{1456}\right)-\left(3 s_{123}+4 s_{144}\right)\left(9 c_{123}+18 c_{144}+8 c_{456}\right)\right] \\
& +\frac{16}{3} s_{44} a_{1}\left[s_{144}\left(3 c_{144}+4 c_{456}\right)-\frac{8}{3} s_{44}^{2} c_{1456}\right], \\
s_{1255}=- & \frac{4}{3} a_{1}^{2}\left[s_{44}^{2}\left(3 c_{1255}+2 c_{1456}+2 c_{4455}\right)-\frac{1}{12}\left(3 s_{144}+4 s_{456}\right)\left(9 c_{123}+18 c_{144}+8 c_{456}\right)\right] \\
& +\frac{16}{3} a_{1} s_{44}\left[s_{44}^{2} c_{1456}-\frac{1}{6}\left(3 s_{144}-s_{456}\right)\left(3 c_{144}+4 c_{456}\right)\right] \\
& +\frac{32}{3} s_{44}^{4} c_{4455}+\frac{8}{9} s_{44}^{2}\left(9 c_{144} s_{144}+12 c_{144} s_{456}+8 c_{456} s_{456}\right), \\
s_{1456}=- & 4 a_{1} s_{44}\left[2 s_{44}^{2} c_{1456}+s_{456}\left(3 c_{144}+4 c_{456}\right)\right], \\
s_{4455}=- & 16 s_{44}^{4} c_{4455}-4 s_{44}^{2}\left(3 c_{144} s_{144}+4 c_{144} s_{456}+4 c_{456} s_{144}+8 c_{456} s_{456}\right),
\end{aligned}
$$

Acknowledgements The authors would like to thank...

\section{References}

1. Saccomandi G, Vergori L (2021) Some remarks on the weakly nonlinear theory of isotropic elasticity. J Elast DOI https://doi.org/10.1007/s10659-021-098651

2. Kostek S, Sinha BK, Norris AN (1993) Third-order elastic constants for an inviscid fluid. J Acoust Soc Am 94:3014-3017, DOI https://doi.org/10.1121/1.407336

3. Hamilton MF, Iliinskii YA, Zabolotskaya (2004) Separation of compressibility and shear deformation in the elastic energy density (1). J Acoust Soc Am 116:41-44, DOI 10.1121/1.1736652

4. Ogden RW (1974) On isotropic tensors and elastic moduli. Proc Camb Phil Soc 75:427-436, DOI https://doi.org/10.1017/S0305004100048635

5. Gennisson JL, Rénier M, Catheline S, Barrière C, Bercoff J, Tanter M, Fink M (2007) Acoustoelasticity in soft solids: assessment of the nonlinear shear modulus with the acoustic radiation force. J Acoust Soc Am 122:3211-3219, DOI 10.1121/1.2793605 
6. Gennisson JL, Deffieux T, Fink M, Tanter M (2013) Ultrasound elastography: Principles and techniques. Diagn Interv Imaging 94:487-495, DOI https://doi.org/10.1016/j.diii.2013.01.022

7. Destrade M, Ogden RW (2010) On the third- and fourth-order constants of incompressible isotropic elasticity. J Acoust Soc Am 128:3334-3343, DOI 10.1121/1.3505102

8. Destrade M, Martin P, Ting T (2002) The incompressible limit in linear anisotropic elasticity, with applications to surface waves and elastostatics. J Mech Phys Sol 50:1453-1468, DOI https://doi.org/10.1016/S00225096(01)00121-1

9. Brugger K (1965) Pure modes for elastic waves in crystals. J Appl Phys 36:759768, DOI 10.1063/1.1714215

10. Norris AN (1998) Finite-amplitude waves in solids. In: Hamilton MF, Blackstock D (eds) Nonlinear Acoustics, Academic Press, San Diego, pp 263-278

11. Landau LD, Lifshitz EM (1986) Theory of elasticity. In: Course of Theoretical Physics Volume 7, 3rd edn, Butterworth-Heinemann, Oxford, pp 1-186

12. Kube CM (2017) Scattering of harmonic waves from a nonlinear elastic inclusion. J Acoust Soc Am 141:4756, DOI https://doi.org/10.1121/1.4986747 